\title{
NORMAS Y PRINCIPIOS DE AUDITORÍA APLICABLES EN LA \\ EVIDENCIA Y PAPELES DE \\ DE TRABAJO
}

CPC. ELMER ZANABRIA HUISA

\section{RESUMEN}

Previamente señalaré que las Normas y Principios de Auditoría son los lineamientos básicos que el auditor debe seguir en el cumplimiento de su labor de auditoría.

El trabajo de algunas firmas de auditoría y de los auditores independientes ha sido y seguirá siendo objeto de severas críticas por parte de los usuarios y de la ciudadanía en cuanto al tema "obtención de evidencia y formulación de papeles de trabajo". Este problema se acentúa por el desconocimiento e inobservancia de las normas de auditoría y procedimientos aplicados para sustentar o fundamentar el informe corto y largo de auditoría. La omisión de estos requisitos durante el proceso de auditoría implica responsabilidades de carácter civil y penal.

El objetivo del presente, ha sido analizar la aplicación de NAGAs (Normas de Auditoría Generalmente Aceptadas); SAS (Declaraciones sobre Normas de Auditoría; NIAs (Normas Internacionales de Auditoría) y NAGU (Normas de Auditoría Gubernamental); NICs (Normas Internacionales de Contabilidad) y FASB (Normas de Contabilidad Financiera) relacionados con la obtención de evidencia y preparación de papeles de trabajo de auditoría.

¿Qué NAGAs, SAS, NIAs, NAGU, NICs y FASB son aplicables a la evidencia y papeles de trabajo de auditoría? y ¿cuál es su utilidad? Para responder estas interrogantes se han planteado las siguientes hipótesis:

- Las NAGAs, SAS, NIAs y NAGU, son aplicables en la obtención de la evidencia y preparación de los papeles de trabajo para fundamentar la opinión o dictamen del auditor independiente.

- Las NICs y FASB tienen aplicación en la formulación de los estados financieros, que es responsabilidad de la gerencia de la empresa.

La metodología utilizada en este trabajo fue el método descriptivo e inductivo, bajo el procedimiento de visita a instituciones, recopilación de la información y consulta bibliográfica; planteamiento del esquema, análisis y desarrollo de los temas.

\section{1.- EVIDENCIA DE AUDITORIA}

1.1 Concepto.- La evidencia de auditoría es la información que obtiene el auditor para extraer conclusiones en las cuales sustenta su opinión. ${ }^{1}$

1.2 Características de la evidencia.- La evidencia obtenida por el auditor debe reunir las siguientes características:

(1) NIA 8 "Evidencia de Auditoría”, p. 227. 


\section{CONTROL DEL COSTO DE IMPORTACIÓN $\quad \mathbf{N}^{\circ}$............}

Requisición $\mathrm{N}^{\circ}$......... O/C $\mathrm{N}^{\circ}$.

Artículo.

Código $\mathrm{N}^{\mathrm{o}}$.

Proveedor

País/localidad

Crédito documentario $\mathrm{N}^{\circ}$.

Importe.

Banco local.

Transporte.

..Lugar de embarque/desembarque

Fecha de embarque/desembarque

Ingreso al almacén: Fecha.

Ag. de aduana

Estado de recepción:

Completo Incompleto Bueno Dañado

\begin{tabular}{|c|c|c|c|c|c|}
\hline $\begin{array}{l}\text { LIQUIDACIÓN: } \\
\text { Detalle }\end{array}$ & Fecha & Compte. $\mathbf{N}^{\mathbf{o}}$ & ME US\$ & $\mathrm{TCV}$ & $\mathrm{MN}$ \\
\hline FOB & & a.... & ..... & & \\
\hline Flete.............................................. & ............. & ................. & ............... & $\ldots$ & \\
\hline Seguro............................................. & ........... & ................. & ............... & ............... & .............. \\
\hline 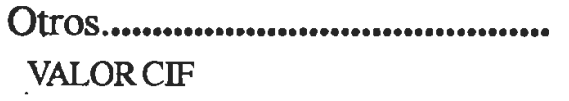 & .............. & ...es.............. & 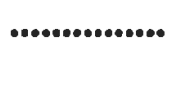 & ............... & ................ \\
\hline
\end{tabular}

(+) COSTOS ADICTONALES:

Gastos de despacho:

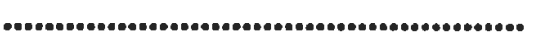

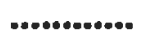

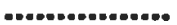

-................

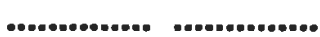

Impuestos:

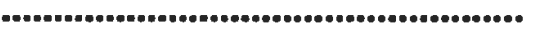

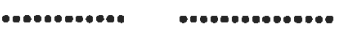

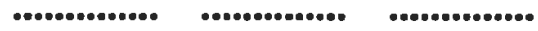

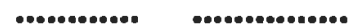

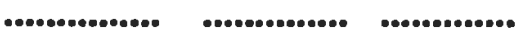

Gastos bancarios y otros:

๑

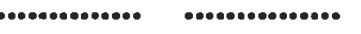

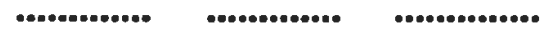

COSTO ACUMULADO

COSTOUNTTARIO

GASTOS/DESEMBOLSOS NO

COSTEABLES:

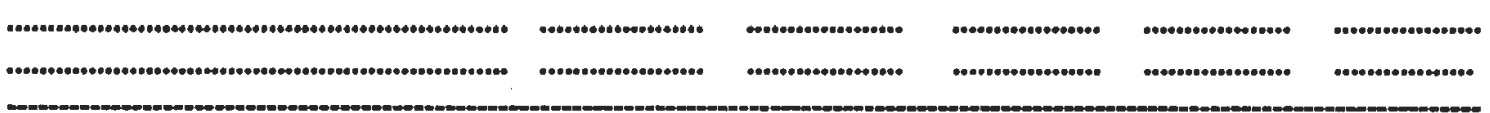

OBSERVACIONES:

Lima,..... de.................de........

Hecho por Revisado Autorizado 
- Competencia.- Es la "medida de la calidad de evidencia de la auditoría y su relevancia para una particular afirmación y su confiabilidad". La evidencia será más confiable cuando se base en hechos más que en criterios.

- Suficiencia.- Es la "medida de la cantidad de evidencia de la auditoría". El auditor, a su criterio profesional, obtiene evidencia suficiente al tener en cuenta los factores como: posibilidad de información errónea, importancia y costo de la evidencia.

Una evidencia se considera competente y suficiente si cumple las características siguientes:

- Relevancia.-La evidencia es relevante cuando ayuda al auditor a llegar a una conclusión respecto a los objetivos específicos de auditoría.

- Autenticidad.- La evidencia es auténtica cuando es verdadera en todas sus características.

- Verificabilidad.- Es el requisito de la evidencia que permite que dos o más auditores lleguen por separado a las mismas conclusiones, en iguales circunstancias. $\mathrm{Si}$ diferentes auditores llegaran a distintas conclusiones examinando el mismo asunto, entonces no habría el requisito de verificabilidad.

- Neutralidad.- Es el requisito que esté libre de prejuicios. Si el asunto bajo estudio es neutral, no debe haber sido diseñado para apoyar intereses especiales.

Para obtener la cantidad de evidencia necesaria y de calidad, el auditor normalmente aplica pruebas selectivas o de muestreo estadístico a la información disponible, no examina toda la información; de esta manera se puede llegar a conclusio- nes sobre un saldo de una cuenta, clases de transacciones o control.

La obtención de evidencia suficiente y competente en la auditoría es afectada por factores como:

- La evaluación de la naturaleza y nivel del riesgo inherente, del giro del negocio, situación económica y financiera de la entidad. Cuanto mayor sea el nivel de riesgo inherente mayor será la cantidad de evidencia necesaria.

- Evaluación de riesgos de control, así como de los sistemas de contabilidad y de control interno.

- Materialidad de la partida o transacción que se examina. previas.

- Experiencia obtenida en auditorías

- Fuente y confiabilidad de información disponible.

\subsection{Obtención de la evidencia en} auditoría.- El auditor debe obtener evidencia suficiente y competente mediante la aplicación de pruebas y procedimientos sustantivos y/o métodos (técnicas) para fundamentar su opinión y conclusiones sobre los estados financieros y otras actividades materia de la auditoría.

a) Pruebas de control.- Se realizan con el objeto de obtener evidencia sobre la idoneidad del sistema de control interno y contabilidad.

b) Pruebas sustantivas.- Consiste en examinar las transacciones y la información producida por la entidad bajo examen, aplicando los procedimientos de auditoría, con el objeto de validar las afirmaciones y 
para detectar las distorsiones materiales contenidas en los estados financieros.

Las afirmaciones son manifestaciones o declaraciones de la gerencia respecto a los estados financieros; es decir, sobre la situación patrimonial (activo, pasivo y capital), los resultados de las operaciones y los cambios en la situación financiera de la entidad auditada. Estas afirmaciones se someten a prueba por el auditor. El SAS $N^{\circ} 31$ establece los siguientes criterios para evaluar tales afirmaciones:

- Existencia u ocurrencia

- Integridad

- Derechos y obligaciones

- Valuación o aplicación

- Presentación y revelación

Las evidencias deben guardar relación con los objetivos de la auditoría y las declaraciones de la gerencia. Por ejemplo, al examinar las afirmaciones de la partida $o$ componente de existencias del balance general efectuada por la gerencia, se debe comprobar la existencia en almacenes, integridad de los saldos, propiedad; método y los procedimientos aplicados en su valuación y los costos de este rubro.

La tercera norma de ejecución del trabajo exige que debe obtenerse evidencia suficiente y competente mediante la inspección, observación, investigación y confirmación para tener una base razonable y poder expresar una opinión respecto a los estados financieros que se examinan. ${ }^{2}$

Esta norma guía al auditor en el desarrollo de los objetivos específicos de auditoría de cada etapa y en la determinación de la competencia de la evidencia, su suficiencia y el método apropiado para obtenerla.

(2) NAGA 3 "Evidencia suficiente y competente" Normas de Ejecución del Trabajo de Auditoría.
La mayor parte del trabajo del auditor independiente, al formular su opinión sobre los estados financieros, consiste en obtener y evaluar la evidencia relativa a las afirmaciones hechas en esos estados financieros. La medida de la validez de dicha evidencia para fines de auditoría descansa en el juicio del auditor, a este respecto la evidencia en auditoría difiere de la evidencia legal, la cual está circunscrita por reglas rígidas. ${ }^{3}$

El auditor obtiene evidencia mediante la aplicación de procedimientos de inspección y procedimientos analíticos; sin embargo, la oportunidad de éstos dependerá de los periodos en que la evidencia buscada se encuentra. ${ }^{4}$

La NAGU 3.40 indica que el auditor debe obtener evidencia suficiente, competente y relevante, mediante la aplicación de pruebas de control y procedimientos sustantivos que le permitan fundamentar razonablemente los juicios y conclusiones que formule respecto de la auditoría. La evidencia deberá someterse a prueba para asegurar que cumpla los requisitos básicos de suficiencia, competencia y relevancia. ${ }^{5}$

Los procedimientos y/o métodos a que hacen referencia las normas indicadas se explican a continuación:

\subsubsection{Métodos y procedimientos para} obtener evidencia.- Los métodos se conocen como técnicas de auditoría utilizados por el auditor para obtener evidencia.

Los procedimientos explican cómo se aplican esas técnicas en el proceso de obtención de evidencia. ${ }^{6}$

(3) Declaraciones sobre Normas de AuditoríaSAS-31.

(4) NIA 8 "Evidencia de Auditoría”.

(5) NAGU 3.4 "Evidencia suficiente, competente y relevante".

(6) J.W. Cook "Auditoría". 
El auditor debe obtener evidencia en la auditoría por uno o más de los siguientes procedimientos: Inspección (documental y examen físico), observación, entrevista o interrogatorio, confirmación, cálculo, procedimientos analíticos y comparaciones.

a. Inspecciones.- Comprende la inspecpección documental y examen físico.

Inspección documental.- Consiste en examinar registros, documentos o activos tangibles. La inspección de registros y documentos proporciona evidencia en la auditoría de grados variables de confiabilidad dependiendo de su naturaleza y fuente y de la efectitividad de los controles internos sobre su procesamiento.

Examen físico.- Es la técnica de la auditoría mediante el cual el auditor verifica la existencia de los activos tangibles para obtener evidencia en la auditoría con respecto a su inclusión en el activo, pero no necesariamente a su propiedad o valor.

b. Observación.- Consiste en presenciar un proceso o procedimientos que están siendo realizados por otros; por ejemplo, la observación del auditor del conteo de inventarios por personal de la entidad o el desarrollo de procedimientos de control.

\section{c. Indagación mediante entrevista.-} Consiste en buscar la información adecuada, dentro o fuera de la organización del cliente. Las indagaciones pueden ser por escrito y en forma oral, y deben documentarse.

d. Confirmación.- Es la respuesta a una pregunta o solicitud, para comroborar la información obtenida en los registros contables, por ejemplo la circularización para bancos comerciales, cuentas por cobrar de clientes, entre otros, etc. para confirmar los saldos vigentes que aparecen en los estados financieros.

Las confirmaciones pueden ser orales o escritas; las manifestaciones por escrito, bajo la forma de carta de manifestaciones dirigidas al auditor, generalmente estarán firmadas por funcionarios responsables.

e. Cálculo.- Consiste en comprobar directamente la exactitud aritmética de los documentos de origen y de los registros contables, o desarrollar cálculos independientes comprobatorios.

f. Procedimientos analíticos.- Consisten en utilizar los índices y tendencias significativas para determinar la liquidez, posición financiera, capacidad de endeudamiento, rentabilidad y gestión empresarial; así como, las fluctuaciones y relaciones inconsistentes en los componentes del balance general y estado de resultados. A través de esta información se lleva a cabo una adecuada planificación y desarrollo de la auditoría. Estos procedimientos proporcionarán una fuente importante de evidencia durante la auditoría y constituirán una parte importante de la evaluación de la racionalidad general de los estados financieros cuando nos formemos una opinión sobre tales estados.

g. Comprobación.- El auditor dedica la mayor parte de la auditoría a los asuntos donde es mayor la posibilidad de error o información equivocada. Un método para determinar estas áreas es la comparación.

\subsubsection{Confiabilidad y fuentes de evi-} dencia en auditoría.- Para que la evidencia de auditoría sea aceptable debe ser confiable y estar influenciada por la fuente (externa e interna) y forma como se obtiene (visual, documentos u oral). Los siguientes criterios ayudarán a evaluar la confiabilidad de la evidencia en la auditoría: 
- La evidencia obtenida de fuentes externas es de mayor confiabilidad que la obtenida dentro la empresa. Por ejemplo, las confirmaciones recibidas de una tercera persona es más confiable que la generada internamente.

- La evidencia generada internamente es más confiable cuando los sistemas de contabilidad y de control interno relacionados son efectivos.

- La evidencia obtenida directamente por el auditor es más confiable que la obtenida en la propia entidad.

- La evidencia en la auditoría en forma de documentos y manifestaciones escritas es más confiable que las manifestaciones orales. $^{7-8}$

La evidencia proveniente de Sistemas Computarizados. ${ }^{9}$

Cuando la información procesada por medios electrónicos constituya una parte importante o integral de la auditoría y su confiabilidad sea esencial para cumplir los objetivos del examen, el auditor deberá cerciorarse de la relevancia y de la confiabilidad de esa información.

Para determinar la confiabilidad de la información, el auditor:

- Podrá efectuar una revisión de los controles generales de los sistemas computarizados y de los controles relacionados específicamente con sus aplicaciones, que incluya todas las pruebas que sean permitidas.

(7) Seminario Regional Interamericano de Contabilidad-Arequipa Oct. 1996

(8)(9) NAGU 3.40 "Evidencia Suficiente, Competente y Relevante".
- Si no se revisa los controles generales y los relacionados con las aplicaciones o se comprueba que esos controles no son confiables, podrá practicar pruebas adicionales o emplear otros procedimientos.

Cuando el auditor utilice datos procesados por medios electrónicos o los concluya en su informe a manera de antecedentes o con fines informativos, por no ser significativos para los resultados de la auditoría, bastará generalmente que en el informe se cite la fuente de esos datos para cumplir las normas relacionadas con la exactitud e integridad de su informe.

\subsubsection{Clasificación de la evidencia}

a.- Evidencia física.- Se obtiene mediante inspección y observación directa de actividades, bienes o sucesos. La evidencia de esta naturaleza puede presentarse en forma de memorándum (donde se resume los resultados de la inspección o de la observación), fotografías, gráficos, mapas o muestras materiales.

b.- Evidencia documental.- Consiste en información elaborada, como la contenida en cartas, contratos, registros de contabilidad facturas y documentos de la administración, relacionados con su desempeño.

c.- Evidencia testimonial.- Se obtiene de otras personas en forma de declaraciones hechas en el curso de indagaciones o entrevistas. Las declaraciones que sean importantes para la auditoría deberán corroborarse, siempre que sea posible, mediante evidencia adicional. También será necesario evaluar la evidencia testimonial para cerciorarse que los informantes no hayan estado influidos por prejuicios o tuvieran solo un conocimiento parcial del área auditada.

d.- Evidencia analítica.- Comprende cálculos, comparaciones, razonamientos, sepa- 
ración de la información en sus componentes.

\section{2.- PAPELES DE TRABAJO}

2.1 Concepto.- Son el conjunto de cédulas y documentos en el cual el auditor registra los datos y la información obtenida durante su examen, los resultados de las pruebas realizadas y la descripción de las mismas.

Son registros que mantiene el auditor de los procedimientos aplicados, pruebas desarrolladas, información obtenida y conclusiones pertinentes a que se llegó en el trabajo de auditoría. ${ }^{10}$

Los papeles de trabajo también pueden constituir la información almacenada en cintas, películas u otros medios (diskettes).

\subsection{Propósito de papeles de trabajo. - Los} papeles de trabajo tienen los siguientes propósitos:

1) Ayudan en la planeación y desarrollo de la auditoría.

2) Ayudan en la supervisión y revisión del trabajo de auditoría.

3) Registran la evidencia en la auditoría para respaldar el informe del auditor.

4)Permiten organizar y coordinar las diferentes fases de auditoría que se lleva a cabo.

5) Sirven de evidencia legal. Los papeles de trabajo constituyen una forma de evidencia que se puede utilizar en acciones legales.

(10) Declaraciones sobre normas de auditoría SAS No 41
Los auditores utilizan los papeles de trabajo para apoyar su conclusión de que realizaron la auditoría de un modo profesional, que ejercieron el debido cuidado y que no fueron negligentes en el desempeño de sus deberes y funciones.

\subsection{Forma y contenido de los papeles}

de trabajo- El auditor al preparar los papeles de trabajo deberá evitar el exceso en la documentación, es decir medir el trabajo por la calidad y no por la cantidad, de tal manera que resulten suficientemente completos y detallados para proporcionar una comprensión global de la auditoría.

En los papeles de trabajo se registran la planeación, la naturaleza, oportunidad y el alcance de los procedimientos de auditoría desarrollados, los resultados, las conclusiones extraídas y las evidencias obtenidas. Incluyen sólo asuntos importantes que se requieran.junto con la conclusión del auditor y los hechos que fueron conocidos por el auditor durante el proceso de auditoría.

La NIA 9 "Documentación" señala que la extensión de los papeles de trabajo es un caso de juicio profesional por lo que es necesario y práctico documentar todos los asuntos importantes que el auditor considere. Igualmente indica que la forma y contenido de los papeles de trabajo es afectada por los factores como: La naturaleza del trabajo de auditoría, el tipo de dictamen o informe del auditor, la naturaleza y evaluación de los sistemas de contabilidad y control interno de la entidad; las necesidades en las circunstancias particulares, de dirección, supervisión, y revisión de los trabajos realizados por los auxiliares; metodología y tecnología de auditoría usadas en el curso del examen.

Los papeles de trabajo son diseñados para cumplir con las circunstancias y las 
necesidades del auditor para cada auditoría en particular. Los papeles de trabajo facilitan la delegación de funciones, y al mismo tiempo proporcionan un medio para control de su calidad.

La SAS- 41 y NIA 9 indican que los papeles de trabajo incluyen, entre otros, las siguientes informaciones:

- Información referente a la estructura orgánica de la entidad examinada.

- Extractos o copias de documentos legales importantes, convenios, y estatutos.

- Información concerniente al entorno económico y legislativo dentro de los que opera la entidad.

- Evidencia del proceso de planeamiento, incluyendo programas de auditoría y cualquier cambio al respecto.

- Evidencia de la comprensión de los sistemas de contabilidad y de control interno.

- Evidencia de evaluaciones de los riesgos inherentes y de control.

- Evidencia sobre la evaluación del trabajo de auditores internos y las conclusiones alcanzadas.

- Evidencia de que los trabajos realizados por los auxiliares fue supervisado y revisado.

-Análisis de transacciones y balances.

-Análisis de tendencias e índice importantes.

-Un registro de la naturaleza, tiempo y grado de los procedimientos de auditoría desarrollados y de los resultados de dichos procedimientos.

-Una indicación sobre quien desarrolló los procedimientos de auditoría y cuando fueron desarrollados.

-Copias de comunicaciones con otros auditores, expertos y otras terceras partes.

- Copias de cartas o notas referentes a asuntos de auditoría comunicados, o discutidos con la entidad, incluyendo los términos del trabajo y las debilidades sustanciales en control interno.

- Cartas de presentación recibidas de la entidad.

- Conclusiones alcanzadas por el auditor concernientes a aspectos importantes de la auditoría, incluyendo cómo se resolvieron los asuntos excepcionales o inusuales, revelados por los procedimientos del auditor.

- Copias de los estados financieros, dictamen u otros informes del auditor, etcétera.

2.4 Propiedad, custodia y archivo de los papeles de trabajo.- Los papeles de trabajo son de propiedad de los órganos conformantes del Sistema Nacional de Control y de las Sociedades de Auditoría cuyos exámenes practicados contaron con la autorización de la Contraloría General.El auditor debe guardar con cuidado y vigilancia la integridad de los papeles de trabajo, debiendo asegurar en todo momento, y bajo cualquier circunstancia, el carácter secreto de la información contenida en los mismos.

Es difícil establecer el tiempo que un auditor debe conservar los papeles de trabajo, pero es recomendable conservarlos porque son importantes para auditorías 
futuras y para cumplir con los requerimientos legales en caso de litigios.

Los archivos de papeles de trabajo para cada examen pueden dividirse en dos grupos básicos: Archivos corrientes y archivos permanentes.

Los archivos corrientes contiene las informaciones relacionadas con la planificación y supervisión que no son de uso continuo en auditorías posteriores tales como: Revisiones corrientes de controles administrativos, análisis de información financiera, correspondencias corrientes, programas de auditoría y otros papeles que respaldan las observaciones y preparación del informe, inclusive el borrador del informe.

Los archivos permanentes deberán contener informaciones importantes para utilizar en auditorías futuras tales como: El historial legislativo sobre la creación de la entidad y sus programas y actividades, la legislación de aplicabilidad continua en la entidad, políticas y procedimientos de la entidad, financiamiento, organización y personal, políticas y procedimientos de presupuestos, contabilidad e informes, estatutos, memorias anuales, etc.

\section{3.- LAS NORMAS Y PRINCIPIOS DE CONTABILIDAD Y AUDITORÍA EN RELACION CON LA EVIDENCIA Y PAPELES DE TRABAJO}

Las NICs (Normas Internacionales de Contabilidad) y Principios de Contabilidad Generalmente Aceptadas y las FASB (Normas de Contabilidad Financiera) se aplican para formular, presentar e interpretar en forma correcta la información financiera, de las cuales es responsable la gerencia de la empresa. Los auditores evalúan la aplicación de estas normas a la elaboración de los estados financieros, con la finalidad de emitir su opinión sobre la razonabilidad o no de los mismos. Es decir se verifica si la información financiera bajo examen se ha preparado utilizando las Normas y Principios de Contabilidad Generalmente Aceptados y sí además, fueron aplicados consistentemente.

Por tanto no son aplicables directamente en la evidencia ni en la preparación de papeles de trabajo de auditoría.

Las NICs son los criterios básicos o guías fundamentales que tiene el propósito de lograr la unificación de criterios contables a nivel internacional, en la elaboración, presentación y correcta interpretación de los Estados Financieros.

La metodología para redactar y aprobar estas normas es la siguiente:

1. La redacción esta a cargo del comité de normas internacionales de contabilidad cuya sigla en inglés es IASC.

2. La aprobación corresponde a la Federación Internacional de Contabilidad -IFAC.

3. La IFAC da a conocer las NICs aprobadas a las organizaciones profesionales e Instituciones representativas de cada país a efecto de análisis y sugerencias para su aplicación.

4. Previo un análisis, cada país adopta su aplicación, de acuerdo a su realidad. En caso del Perú se oficializa la aplicación de las NICs por Resolución del Consejo Normativo de Contabilidad.

En nuestro país, desde 1986, han sido puestas en vigencia 33 NICs, previa aprobación en los Congresos Nacionales de Contadores Públicos. A la fecha se encuentran 5 normas más en proceso de análisis para su aplicación, con los cuales serían 38 NICs en total oficializados. 
Las FASB son los Principios de Contabilidad Generalmente Aceptados emitidas por el Comité de Normas de Contabilidad Financiera para la elaboración y presentación de la información financiera aplicable a la realidad de Estados Unidos. Estas normas se toman en cuenta como referencia en nuestro país.

\section{Las Normas y Principios de Auditoría.-} Comprende las normas de auditoría generalmente aceptadas (normas personales, de ejecución de trabajo y preparación del informe), NIAs (Normas Internacionales de Auditoría), SAS (Declaraciones sobre Normas de Auditoría) y NAGU (Normas de auditoría gubernamental).

Las Normas de Auditoría son los lineamientos básicos que el auditor debe seguir en el cumplimiento de la labor de auditoría. El organismo encargado de elaborar las NIAs para la ejecución de tareas de auditoría es el Comité Internacional de Prácticas de Auditoría cuya sigla en inglés es IAPC (International Auditing Practices Comittee) y es aprobado por la IFAC, luego se dan a conocer a las diferentes organizaciones profesionales y reparticiones de cada país a fin de su estudio y sugerencias y de que adopten su aplicación en sus jurisdicciones.

A la fecha están en vigencia las siguientes normas internacionales de auditoría:

NIA 1. Objetivo y alcance de la auditoría de estados financieros.

NIA 2. Carta compromiso de auditoría.

NIA 3. Principios básicos que rige una auditoría.

NIA 4. Planeamiento.

NIA 5. Utilización del trabajo de otro auditor.

NIA 6. Estudio y evaluación del sistema contable y los correspondientes controles internos, en relación con la auditoría.
NIA 7. Control de la calidad del trabajo de auditoría.

NIA 8. Evidencia de auditoría.

NIA 9. Documentación.

NIA 10. Utilización del trabajo de un auditor interno.

NIA 11. Fraude y error.

NIA 12. Procedimientos analíticos.

NIA 13. El Dictamen del auditor sobre los estados financieros.

NIA 14. Otra información en documentos que contienen estados financieros auditados. NIA 15. Auditoría en un ambiente PED (procesamiento electrónico de datos).

NIA 16. Técnicas de auditoría con ayuda del computador.

NIA 17. Entidades vinculadas.

NIA 18. Utilización del trabajo de un experto.

NIA 19. Muestreo de auditoría.

NIA 20. Los efectos del ambiente PED en el estudio y evaluación del sistema de contabilidad y los correspondientes controles internos.

NIA 21. Fecha del dictamen del auditor. Hechos posteriores a la fecha del balance general: Descubrimientos de hechos después de la emisión de los estados financieros.

NIA 22. Manifestación de la gerencia.

NIA 23. Empresa en marcha.

NIA 24. Dictamen del auditor con fines especiales.

NIA 25. Materialidad y riesgo de auditoría. NIA 26. Auditoría de estimados contables. NIA 27. Examen de información financiera preelaborada.

NIA 28. Compromiso de primera auditoría. Saldos de apertura.

NIA 29. Evaluación del riesgo inherente y del riesgo de control y su impacto en los procedimiento sustantivos.

\section{CONCLUSIONES Y RECOMENDACIONES}

La obtención de evidencia suficiente y competente y la adecuada preparación de 
los papeles de trabajo constituyen el soporte fundamental de la opinión de los auditores.

En la obtención de evidencia de auditoría se aplican la 3ra. Norma de Auditoría Generalmente Aceptada referente a la ejecución de trabajo, Declaración sobre Normas de Auditoría N 31, Norma Internacional de Auditoría No 8 "Evidencia" y Normas de Auditoría Gubernamental No 3.40 'Evidencias suficientes, competente y relevante". Estas normas se complementan y se aplican en auditorías de entidades del sector privadoy gubernamental, las Normas de Auditoría Gubernamental están dadas para el sector público.

En la preparación de los papeles de trabajo se aplican la Declaración sobre Normas de Auditoría No. 41, Norma Internacional de Auditoría No 9, 'Documentación" y Normas de Auditoría Gubernamental 3.50 “Papeles de Trabajo". Las Normas de Auditoría Generalmente Aceptadas no hacen referencia a los papeles de trabajo. Los papeles de trabajo son el registro de la información y evidencias obtenidas y las pruebas desarrolladas durante el examen para respaldar la opinión y conclusiones del auditor.

La evidencia y papeles de trabajo se complementan en la labor de auditoría.

Las Normas Internacionales de Contabilidad y Principios de Contabilidad Generalmente Aceptados se aplican en la formulación de los Estados Financieros, de los cuales es responsable la gerencia de la entidad. Los auditores evalúan la aplicación de estas normas en la elaboración, presentación e interpretación de los Estados Financieros bajo examen, y verifican si fueron aplicados consistentemente de un año a otro.

Es recomendable el estudio y análisis más detallado de las NAGAs, SAS, NAGU,
NICs y FASB a nivel internacional a efecto de armonización de éstas en la labor de auditoría.

\section{BIBLIOGRAFÍA}

NAGAs (Normas de Auditoría Generalmente Aceptados).

INSTITUTO INTERAMERICANODE AUDITORIA Y.CONTABILIDAD

NIAs (Las Normas Internacionales de Auditoría), lra.Ed., enero 1996, Lima, Perú.

NAGU (Normas de Auditoría Gubernanamental) aprobado por Resolución de Contraloría No 162 - 95 - CG.

SAS (Declaraciones sobre Normas de Auditoría), emitida por el Comité Ejecutivo de Normas de Auditoría. AICPA, noviembre 1994, México.

\section{J.W. COOK}

"Auditoría". 3ra. Ed. Litográfica Ingramez, enero 1996, México.

YARASCA R., Pedro A.

"Auditoría. Fundamento con un enfoque moderno". Editado en Talleres de $\mathrm{H}$ y R Artes Gráficas SRL, octubre 1995, Lima, Perú.

Seminario Regional Interamericano. "Reinventando la Auditoría" Organizado por CC.PP. Arequipa, octubre - noviembre, 1996.

Manual de Auditoría. Contraloría GeneralCentro de Capacitación de Ciencias Fiscalizadoras, 1983, Lima, Perú. 